\title{
Localized Giant Cell Tumor of the Tendon Sheath: A Rare Case of Anterior Knee Pain
}

\author{
Bairi Cui ${ }^{1}$, Kumar Gurung${ }^{2}$, Yuanming He1, Guo Song1, Longhao Jin ${ }^{1 *}$ \\ ${ }^{1}$ Department of Orthopedic Surgery, Yanbian University Hospital, Yanji, China \\ ${ }^{2}$ Department of Orthopedic Surgery, Community Hospital, Itahari, Nepal \\ Email: *jlh0423@sina.com
}

How to cite this paper: Cui, B.R., Gurung, K., He, Y.M., Song, G. and Jin, L.H. (2019) Localized Giant Cell Tumor of the Tendon Sheath: A Rare Case of Anterior Knee Pain. Journal of Biosciences and Medicines, 7 , 16-21.

https://doi.org/10.4236/jbm.2019.77002

Received: May 5, 2019

Accepted: July 6, 2019

Published: July 9, 2019

Copyright $\odot 2019$ by author(s) and Scientific Research Publishing Inc. This work is licensed under the Creative Commons Attribution International License (CC BY 4.0).

http://creativecommons.org/licenses/by/4.0/

\begin{abstract}
This report describes a 61-year-old female with a giant cell tumor of the tendon sheath (GCT-TS). MRI showed that an elliptical abnormal signal was observed over the infrapatellar region of the right knee. We directly do arthroscopy to remove the tumor. An oval irregular mass of about $2.0 \mathrm{~cm} \times$ $1.5 \mathrm{~cm} \times 1 \mathrm{~cm}$ in the right knee joint was found. It was hard and had a dark red surface and the pedicle of the mass was connected with the joint capsule and infrapatellar fat pad. Nodular GCT-TS occurs less frequently in large joints than the small joints of the fingers and toes. The current report demonstrates the unique characteristics of the GCT-TS that extends around the ankle and invades the knee and proximal humerus.
\end{abstract}

\section{Keywords}

Giant Cell Tumor of the Tendon Sheath (GCT-TS), Knee, Arthroscopy

\section{Introduction}

Giant cell tumor of the tendon sheath (GCT-TS) is a benign tumor of joints, bursa, tendon sheath and synovium, which was first described by Jaffe et al. in 1941 [1]. It is also known as localized nodular tenosynovitis. The incidence of GCT-TS is 1 in $1,800,000$, and most of them grow in the small joints of the hands and feet ( $85 \%$ of cases), rarely found in the knee joints, showing nodular growth [2]. GCT-TS usually occurs in women aged 30 - 50 years [3]. Usually, the patient has no any obvious cause of repeated swelling and pain. GCT-TS is slow in growth, non-aggressive in nature, and few malignant changes may occur. Edge resection is the standard treatment for GCT-TS. However, despite of benign in nature, the rate of local recurrence after resection is reported to range between $10 \%$ and $20 \%$ [3]. The local high recurrence rate may be due to the fact 
that complete resection may be difficult because the mass is often connected to the tendon sheath or synovium.

Here we present a case of GCT-TS involving the infra-patellar fat pad and discuss its clinical and imaging features, differential diagnosis, histopathology and treatment. It can help clinicians build more informed diagnoses and manage the right methods of treatments. Written and informed consent was obtained from the patient.

\section{Case Data}

A 61-year-old female with no obvious cause of right knee pain since one and half year with limited range of motion (ROM), followed by intermittent swelling and paroxysmal pain, and exaggerated while walking upstairs and downstairs. The symptoms were obvious, and occasionally the joints were interlocked during the activity, which got resolved by themselves. On physical examination: the right knee was mildly swollen, with ROM from $0^{\circ}-120^{\circ}$. The patellar tap test was negative, patellofemoral joint tenderness was negative, right knee joint line tenderness was positive, lateral stress test was negative, McMurray test was postive, anterior and posterior drawer test was negative, Lachman test was negative, and no obvious neurovascular deficit was observed. The lab reports were: WBC-5.00 $\times$ $10^{9} / \mathrm{L}, \mathrm{RBC}-3.79 \times 10^{12} / \mathrm{L}, \mathrm{HGB}-118 \mathrm{~g} / \mathrm{L}$, neutrophil count- $2.74 \times 10^{9} / \mathrm{L}$, neutrophil percentage- $54.80 \%$. Chloride ion- $111 \mathrm{mmol} / \mathrm{L}$.

\section{Radiological Findings}

X-ray: No any obvious abnormalities were detected in the radiographs of the right knee (Figure 1(A) and Figure 1(B)).

MRI: An elliptical abnormal signal was observed over the infrapatellar region of the right knee. The $\mathrm{T} 2$ pressure-lowering sequence showed a mixed low signal with a size of about $2.0 \mathrm{~cm} \times 1.0 \mathrm{~cm}$, and the T1WI showed a low signal. The diagnosis was a mass in the right knee joint cavity. Degenerative changes of the meniscus were also noted (Figure 1(C) and Figure 1(D)).

\section{Surgery and Pathology}

Intraoperative findings: An oval irregular mass of about $2.0 \mathrm{~cm} \times 1.5 \mathrm{~cm} \times 1$ $\mathrm{cm}$ in the right knee joint was found. It was hard and had a dark red surface and the pedicle of the mass was connected with the joint capsule and infrapatellar fat pad. One-sixth of the surface of the mass was cut off from the pedicle that was connected to the infrapatellar fat pad of the joint capsule and later on using an arthroscopic blade, it was completely removed.

After removal of the mass, a scalpel was used to cut it from the middle, no blood flow was seen, the cut surface was solid lobulated, irregular curved cracked cavity near the pedicle was visible (Figure 2(A) and Figure 2(B)). After that, the original tumor and the synovial membrane were joined, and a $0.5 \mathrm{~cm}$ synovial membrane was removed with a planing knife. The removed tumor was sent to 


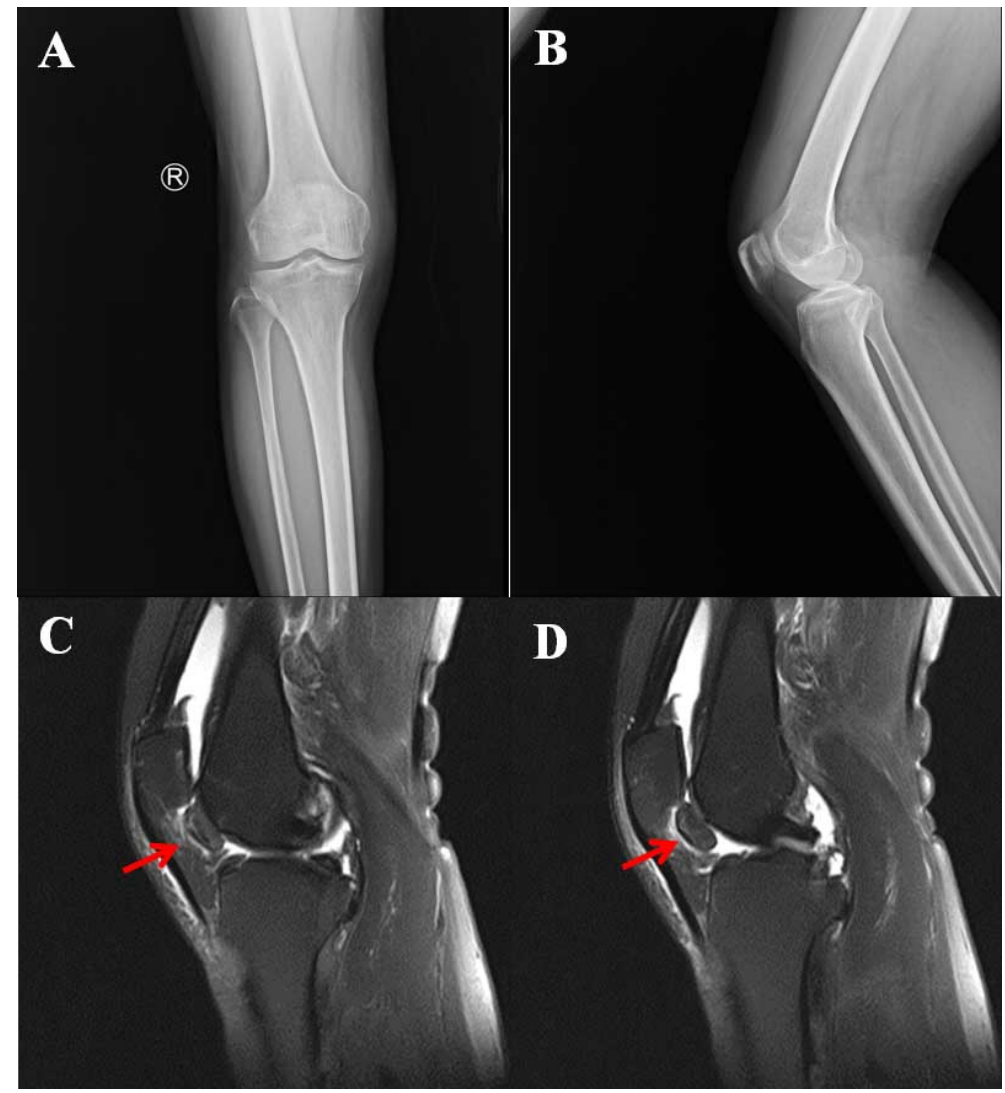

Figure 1. (A) and (B) are X-ray. There was a slight OA manifestation on the inside of the knee joint and no other abnormalities. (C) and (D) are MRI T1 effects. A mass of $2.0 \mathrm{~cm}$ $\times 1.0 \mathrm{~cm}$ on the posterior side of the infra-patellar fat pad was found. The red arrow refers to the mass.

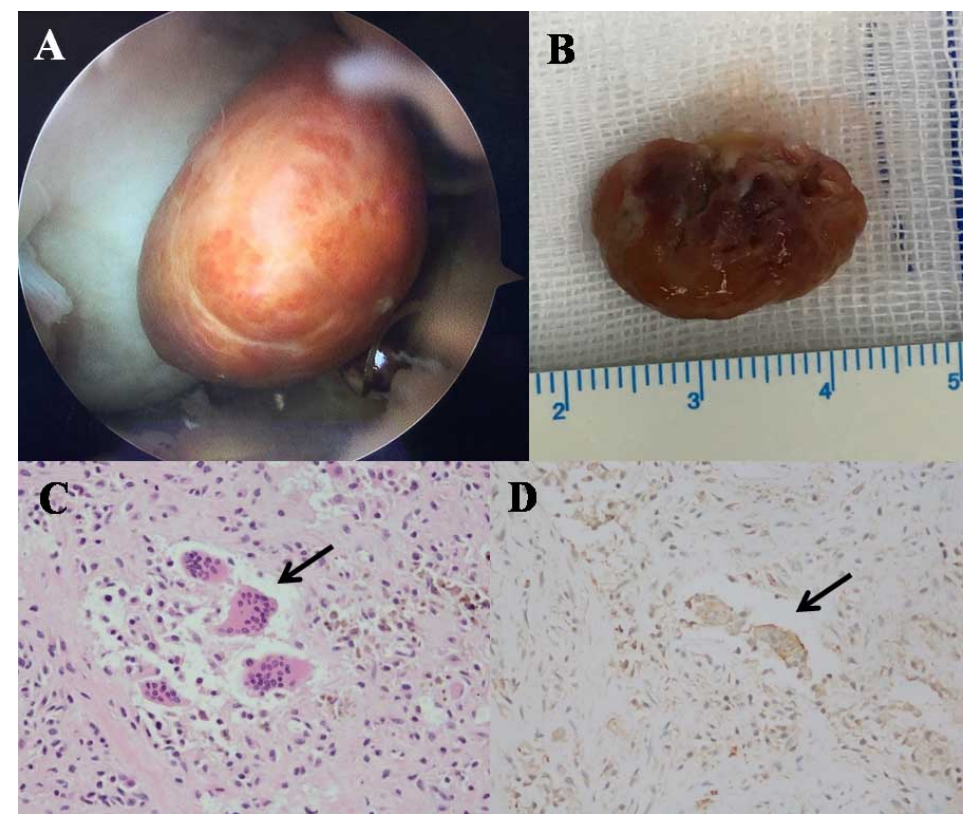

Figure 2. (A) is the mass observed under arthroscopy. (B) is the gross image of the excised mass. (C) is $\mathrm{H} \& \mathrm{E}$ staining image. (D) is CD-68 immunohistochemical staining. Arrows refer to multinucleated giant cells. 


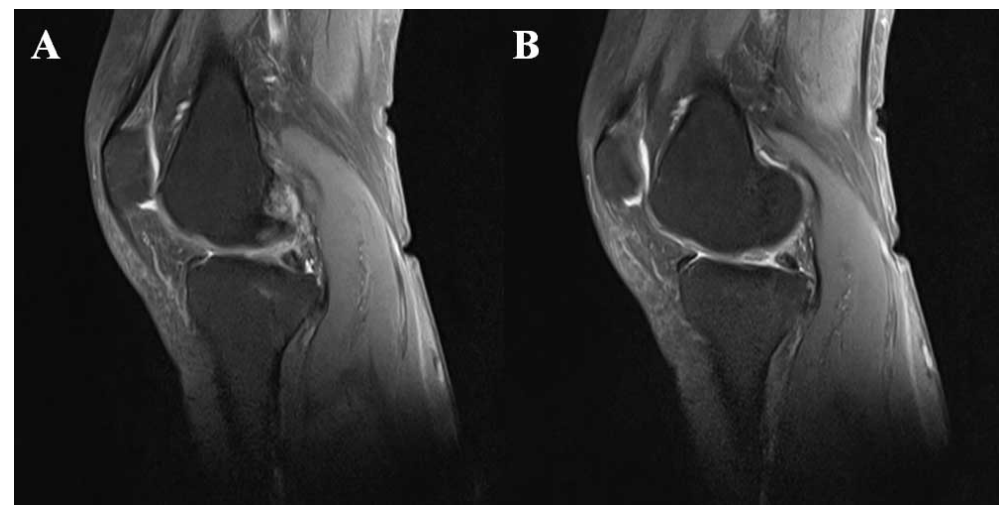

Figure 3. (A) and (B) are MRI T1 effects.

the pathology department.

Histopathological results: Histologically, the lesion contained fibroblasts, tissue cells and fibrous tissue of foam macrophages and dispersed multinucleated giant cells. No mitotic signs, nuclear abnormalities or necrosis were found. CD-68 immunohistochemical staining was positive (Figure 2(C) and Figure 2(D)). According to the radiological and histolopathological findings, the lesion was diagnosed as a giant cell tumor of the tendon sheath.

MRI was performed 10 months after surgery and no recurrence of tumors was found at the site of tumor resection. No tumors were found in other areas (Figure 3(A) and Figure 3(B)).

\section{Discussion}

The localized GCT-TS is macroscopically one or more nodules, brown and yellowish color of different size as per to the hemosiderin. The histopathological features were lobulated leaflets surrounded by dense, transparent collagen. The intratumoral cells are composed of mononuclear cells, osteoblast-like multinucleated giant cells, xanthoma cells, chronic inflammatory cells, hemosiderin macrophages and collagenized matrix in different proportions. CD-68 immunohistochemical staining was positive [2].

Although GCT-TS is histologically identical, it is divided into two different forms according to pattern, diffuse and limited. Local GCT-TS is usually the flexor tendon with extra-articular involvement of the hands and feet. The diffuse form, also known as Pigmented villonodular synovitis (PVNS), is usually intra-articular and involves large synovial joints such as the knee, ankle and shoulder. Diffuse PVNS present as intra-articular hemorrhage in the knee joint whereas the nodular GCT-TS usually manifest as a painless mass [4] [5]. The knee joint usually involves in a diffuse pattern, while the intra-articular GCT-TS is a rare lesion [6].

Although the cause is unclear, more than half of the patients have a history of trauma. The course of the disease progresses slowly, and the interval between the first sign and the diagnosis is longer: 10 months to 3 years. However, acute forms have been reported, related to torsion and necrosis of a pediculated nodule [2]. 
Functional signs are relatively nonspecific, there is joint discomfort in the knee joint, repeated swelling and limited mobility. Locking of the joint, pseudo meniscus symptoms or joint instability maybe felt [7]. During this phase, radiological examination of the knee joint may be helpful for the diagnosis of GCT-TS. The main signs are swelling of the soft tissue around the joint, increased density, and generally no calcification in the soft tissue, with or without compression bone erosion. MRI examination has diagnostic value. On the T2-weighted image, the tumor shows homogenous high-density shadow or peripheral high-density shadow. Due to the presence of hemosiderin, there is no magnetic effect on MRI, the tumor has a fibrous capsule, and the adjacent joint tissue is mostly unaffected.

The standard treatment for GCT-TS is complete resection of the tumor. The most common postoperative complications are local recurrence. Mollon et al. [8] reported that regardless of incision or arthroscopic resection, radiotherapy, the total recurrence rate of all treatments combined was $7.1 \%$.

When the local total resection of the tumor is usually performed, the recurrence rate is low. Residual tumors are the main cause of recurrence. Therefore, Kilicaslan et al. [9] reported that they were more inclined to open surgery to remove the lesion without leaving any residue tissue. However, I recommend arthroscopic resection, because during the arthroscopic operation the image is enlarged, the removal is more comprehensive, with a small trauma and is easy to recover after surgery. On the contrary, the open surgery is not comprehensive, the trauma is large, and the postoperative recovery is slow.

Other treatments are Radiation therapy and targeted therapies. Targeted treatments include anti-TNF alpha, tyrosine kinase inhibitor, a monoclonal antibody inhibiting CSF1receptors [10] [11]. But these treatments apply to diffuse TSGCT or PVNS. The preferred treatment for localized GCT-TS is a complete surgical removal. Additional radiation therapy is also possible.

\section{Conclusion}

In summary, the initial phase of GCT-TS may stimulate the synovium, causing subtle clinical signs and symptoms. Since the X-ray is usually normal and the possibility of neglecting the tumor is high, the doctor is urged not to ignore any minor pain or joint discomfort. In such case, MRI is recommended to rule out soft tissue lesions. Although the tumor may recur, it is recommended to remove it under arthroscopy and to recover well after surgery.

\section{Acknowledgements}

This study was supported by the grant of the National Natural Science Foundation of China (81560353).

\section{Conflicts of Interest}

The authors declare no conflicts of interest regarding the publication of this 
paper.

\section{References}

[1] Jaffe, H.L., Lichtenstein, L. and Sutro, C.J. (1941) Pigmented Villonodular Synovitis, Bursitis and Tenosynovitis. Archives of Pathology, 31, 731-765.

[2] Gouin, F. and Noailles, T. (2017) Localized and Diffuse Forms of Tenosynovial Giant Cell Tumor (Formerly Giant Cell Tumor of the Tendon Sheath and Pigmented Villonodular Synovitis). Orthopaedics \& Traumatology. Surgery \& Research, 103, S91-S97. https://doi.org/10.1016/j.otsr.2016.11.002

[3] Weiss, S.W. and Goldblum, J.R. (2007) Benign Tumors and Tumor-Like Lesions of Synovial Tissues. In: Enzinger and Weiss's Soft Tissue Tumors, 5th Edition, Mosby, St Louis, MO, 769-788.

[4] Relwani, J., Factor, D., Khan, F. and Dutta, A. (2003) Giant Cell Tumor of the Patellar Tendon Sheath-An Unusual Cause of Anterior Knee Pain: A Case Report. The Knee, 10, 145-148. https://doi.org/10.1016/S0968-0160(02)00058-3

[5] Sun, C., Sheng, W., Yu, H. and Han, J. (2012) Giant Cell Tumor of the Tendon Sheath: A Rare Case in the Left Knee of a 15-Year-Old Boy. Oncology Letters, 3, 718-720. https://doi.org/10.3892/ol.2012.555

[6] Lucas, D.R. (2012) Tenosynovial Giant Cell Tumor: Case Report and Review. Archives of Pathology \& Laboratory Medicine, 136, 901-906.

https://doi.org/10.5858/arpa.2012-0165-CR

[7] Dines, J.S., De Berardino, T.M., Wells, J.L., Dodson, C.C., Shindle, M., Di Carlo, E.F., et al. (2007) Long-Term Follow-Up of Surgically Treated Localized Pigmented Villonodular Synovitis of the Knee. Arthroscopy, 23, 930-937. https://doi.org/10.1016/j.arthro.2007.03.012

[8] Mollon, B., Lee, A., Busse, J.W., Griffin, A.M., Ferguson, P.C., Wunder, J.S., et al. (2015) The Effect of Surgical Synovectomy and Radiotherapy on the Rate of Recurrence of Pigmented Villonodular Synovitis of the Knee. An Individual Patient Meta-Analysis. The Bone \& Joint Journal, 97, 550-557. https://doi.org/10.1302/0301-620X.97B4.34907

[9] Kılıçaslan, Ö.F., Katı, Y.A., Kose, O., Erol, B. and Sezgin Alikanoglu, A. (2017) Giant Cell Tumor of the Patellar Tendon Sheath: A Rare Case of Anterior Knee Pain. Cureus, 9, e1690. https://doi.org/10.7759/cureus.1690

[10] Cassier, P., Gelderblom, H., Stacchiotti, S., Thomas, D., Maki, R., Kroep, J., et al. (2012) Efficacy of Imatinib Mesylate for the Treatment of Locally Advanced and/or Metastatic Tenosynovial Giant Cell Tumor/Pigmented Villonodular Synovitis. Cancer, 15, 1649-1656. https://doi.org/10.1002/cncr.26409

[11] Cassier, P., Italiano, A., Gomez-Roca, C.A., Le Tourneau, C., Toulmonde, M., Cannarile, M.A., et al. (2015) CSF1R Inhibition with Emactuzumab in Locally Advanced Diffuse-Type Tenosynovial Giant Cell Tumors of the Soft-Tissue: A Dose-Escalation and Dose-Expansion Phase 1 Study. The Lancet Oncology, 16, 949-956. https://doi.org/10.1016/S1470-2045(15)00132-1 